\title{
Simultaneous exposure to low concentrations of dichlorodiphenyltri- chloroethane, deltamethrin, nonylphenol and phytoestrogens has negative effects on the reproductive parameters in male Spraque- Dawley rats
}

\author{
E. Kilian ${ }^{1,2}$, R. Delport ${ }^{3}$ M. S. Bornman ${ }^{1} \&$ C. de Jager ${ }^{4}$ \\ 1 Department of Urology, University of Pretoria, Pretoria, South Africa; \\ 2 Department of Paraclinical Sciences, Faculty of Veterinary Science, Onderstepoort, South Africa; \\ 3 Department of Chemical Pathology, University of Pretoria, Pretoria, South Africa; \\ 4 School of Health Systems \& Public Health, University of Pretoria, Pretoria, South Africa
}

\section{Summary}

Many reports suggest that male reproductive health has deteriorated over the last decades, possibly due to environmental contaminants that act as endocrine disruptors. This hypothesis was tested in SpragueDawley rats using a modified Organization for Economic Cooperation and Development 415 onegeneration test. Group A received cottonseed oil as control, and Groups B, C and D received deltamethrin (DM); DM and dichlorodiphenyltrichloroethane (DDT); and DM, DDT, phytoestrogens and $p$ nonylphenol, respectively. Rats were exposed in utero and then received the substances for 10 weeks. The seminal vesicle mass (Group B; $P=0.046$ ) and sperm count [Groups $C(P=0.013)$ and $D$ $(P=0.003)$ ] were lower and the anogenital distance [Group B $(P=0.047) \mathrm{C}(P=0.045)$ and $\mathrm{D}$ $(P=0.002)$ ] shorter compared with the control group. The seminiferous tubule diameter [Groups B $(P=<0.001), \mathrm{C}(P=<0.001)$ and $\mathrm{D}(P=<0.001)]$ and epithelium thickness [Groups $\mathrm{B}(P=0.030), \mathrm{C}$ $(P=<0.001)$ and $\mathrm{D}(P=<0.001)]$ were smaller compared with the control. The histology of the testes showed signs of apical sloughing and vacuolisation. Liver weights [Groups $C(P=0.013)$ and $D$ $(P=0.005)$ ] and liver enzymes [Group D $(P=0.013)$ ] were also affected. These findings may indicate that simultaneous exposure to endocrine disrupting compounds contributes to the deterioration observed in male reproductive health.

\section{Introduction}

A variety of oestrogenic compounds exist in the environment, both natural (phyto- and myco-oestrogens) and synthetic (e.g. pesticides, industrial contaminants and sewage effluent by-products). Many of these compounds possess endocrine disrupting effects and it has been suggested that the apparent decline in male reproductive health over the last decades may be caused by exposure to excessive amounts of these oestrogenic compounds used daily in industry, agriculture and in the home (Jensen et al., 1995; Toppari et al., 1996).

Malaria is one of the world's most serious tropical diseases and imposes significant economic burden on some of the poorest nations. Synthetic pyrethroids such as deltamethrin (DM) were developed to limit the use of dichlorodiphenyltrichloroethane (DDT) (Sharp \& le Sueur, 1996). It has been proven that pyrethroids are endocrine disruptors, which can increase the oestrogenic load in the body (Gray et al., 1989) and increase the levels of oestrogen in breast cancer cells (Go et al., 1999; Chen et al., 2002). DM exposure studies showed decreased semen quality and impaired reproductive function in laboratory animals at 1.0 and $2.0 \mathrm{mg} \mathrm{kg}^{-1}$ (El-Aziz et al., 1994). Synthetic pyrethroids, like DM, are currently also being used in agriculture, particularly for cotton-production and in small-scale farming.

Dichlorodiphenyltrichloroethane has been shown to have a negative reproductive effect on the biota (Facemire et al., 1995; Sumpter, 1995). In addition to DDT exposure, exposure to its metabolites also contributes to the detrimental effects on reproduction. Technical-grade DDT (used as an indoor spray) consists of approximately $85 \%$ of $p, p^{\prime}$-DDT, $15 \% o, p^{\prime}$-DDT and trace amounts of $0, o^{\prime}$-DDT (Turusov 
et al., 2002). Both $p, p^{\prime}$-DDT and o,p'-DDT can bind to nuclear oestrogen receptors (ERs) (Charles et al., 2005). p, $p^{\prime}$-dichlorodiphenyl dichloroethylene (DDE), a persistent metabolite of $p, p^{\prime}$-DDT, has been shown to have anti-androgenic activity and to exert developmental toxicities in male rats (Kelce et al., 1995; Gray et al., 2001).

The hypothesis was that $p, p^{\prime}$-DDE may interact in an additive or multiplicative way with other endocrine disrupting chemicals (EDCs). This is particularly important for South Africa, as the vector control programme for malaria uses both DM and DDT for indoor residual spraying, after one of the mosquito vectors that was previously eliminated by DDT, returned and has pyrethroid resistance (Hargreaves et al., 2000). The acceptable daily intake (ADI) for DDT in human milk was set at $20 \mathrm{\mu g} \mathrm{kg}^{-1}$ body weight (bw) and the ADI for DM is set at $10 \mathrm{\mu g} \mathrm{kg}^{-1}$ bw (Bouwman et al., 2006). In their study Bouwman et al. (2006) calculated that the ADI of DDT for infants is exceeded by 1.9 times via breast milk, while the ADI for DM was exceeded slightly at $13.3 \mathrm{\mu g} \mathrm{kg}^{-1} \mathrm{bw}$. It is also important to take into account that the infants are exposed to these levels for up to 2 years, as breast milk constitutes the main food source to these infants (Bouwman et al., 2006).

Humans are also exposed daily to many other EDCs. $p$-Nonylphenol ( $p$-NP) belongs to a group of alkylphenols polyethoxylates and is used in detergents, paints, herbicides, pesticides and cosmetics. $p$ NP was reported to have oestrogenic properties and it was evident from reproductive toxicology studies that it exerted negative effects on the testis and epididymis of rodents (De Jager et al., 1999a,b; Lee et al., 1999). In South Africa, drinking water tested positively for $p$-NP and significant levels were found in sediment and in fatty tissue of eland and fish (Barnhoorn et al., 2002; De Jager et al., 2002). This confirmed that wildlife in South Africa is being exposed to environmental EDCs.

Phytoestrogens are natural compounds present in plants and are ingested daily in milligram quantities. The active substances are isoflavones (genistein), coumestans (coumesterol) and fungal metabolites (mycotoxins), such as zearalenone. Phytoestrogens possess oestrogenic effects in vitro and in vivo and have been responsible for reproductive disorders in some animal species (Toppari et al., 1996; Santti et al., 1998). Neonatal exposure of genistein is reported to inhibit the growth and proliferation of testicular cells in rats (Diaka et al., 1998).

These reports support the concerns that EDCs and combinations of EDCs may have implications for the general and reproductive health of humans and animals or its progeny as a result of changes in endocrine function. In this study, potential adverse effects of DM and combinations of DM, DDT, $p$-NP and phytoestrogens on the male reproductive function and liver, after maternal and lifetime exposures were investigated.

\section{Materials and Methods}

\section{Test system and experimental design}

The study was performed after approval by the Ethics Committee of the University of Pretoria (Project no: 11/2001). The Organization for Economic Cooperation and Development (OECD) 415 protocol for Toxicity Studies was modified to accommodate one control and three experimental groups (OECD Guidelines for the Testing of Chemicals, 1983). The design allowed studying the endocrine disrupting effects of maternal exposure (P1) to known EDCs on the reproductive parameters in lifetime exposed F1 males.

Forty-eight adult Sprague-Dawley rats, 24 males and 24 females, were obtained from The National Health Laboratory Service. They were housed in standard polycarbonate rat cages in rooms with constant temperatures $\left(22 \pm 2{ }^{\circ} \mathrm{C}\right)$, constant humidity $(55 \pm 10 \%)$ and 12-h day/night cycles. They were maintained on a stock pellet diet (Epol mice cubes, Pretoria, South Africa) and had free access to food and tap water. The animals were given a 2-week acclimatisation period before the study started. 
Animals were randomly allocated into one control and three experimental groups and therefore consisted of six breeding pairs each (De Jager et al., 1999a,b). The P1 females were allowed to mate and were observed for vaginal plugs and this was used as day one (D1) of pregnancy. To avoid interference with the blastocyst implantation and embryonic growth, dosing of the P1 females started only on day 7 of pregnancy and continued throughout the gestational and lactational period until the pups were weaned at 23 days.

Twelve F1 male offspring of each group were randomly selected. The individual animals were kept in separate cages and were directly exposed to the substances by oral gavage for a 10-week period. At week 14, the F1 males were terminated for sample collection.

1. Gestational + lactational exposure

P1 females

\begin{tabular}{|c|c|c|c|}
\hline$\underset{\text { (Cottonseed oil) }}{\downarrow}$ & $\begin{array}{c}241 \\
7 \text { day } \\
\downarrow \\
\text { Gr B } \\
\text { (DM) } \\
\text { Gestati } \\
\text { F1 off }\end{array}$ & $\begin{array}{l}\text { mated } \\
\text { nant } ᄋ \\
\downarrow \\
\text { Gr C } \\
\text { (DM + DDT) } \\
\text { Lactation } \\
\text { weaned }\end{array}$ & $\begin{array}{c}\downarrow \\
\text { Gr D } \\
(\mathrm{DM}+\mathrm{DDT}+\mathrm{p}-\mathrm{NP} \\
+ \text { phytoestrogens })\end{array}$ \\
\hline
\end{tabular}

2. Direct exposure

F1 males

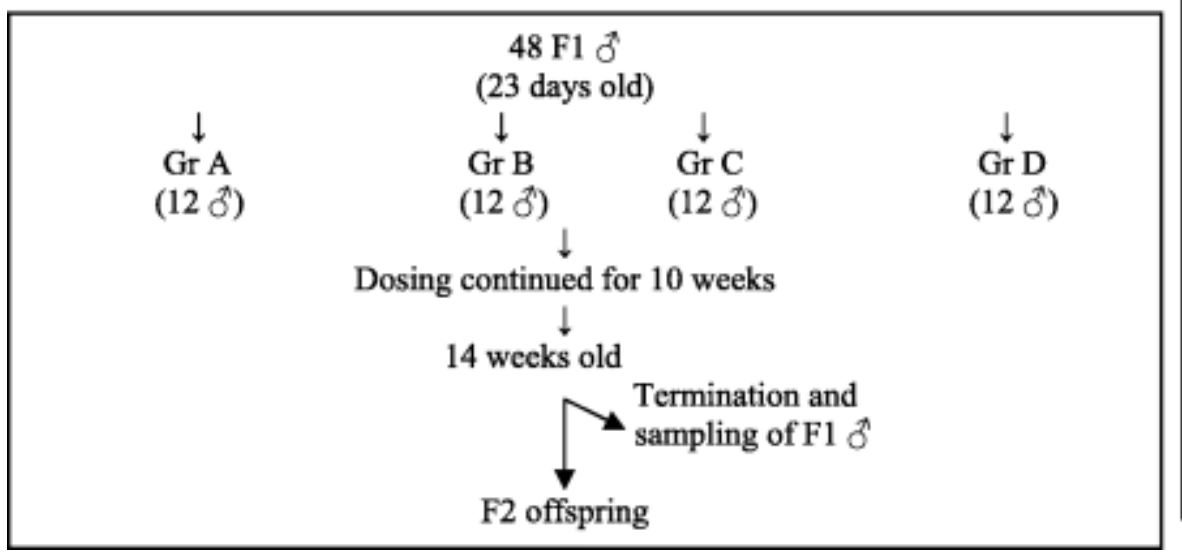

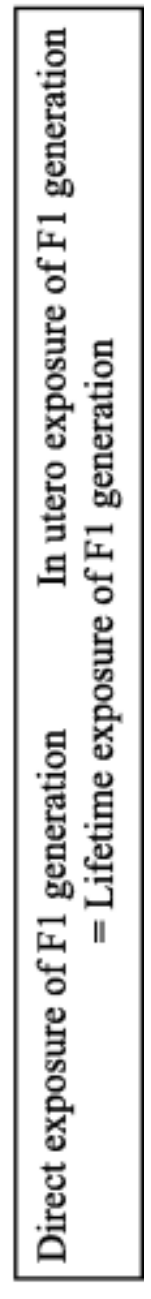

\section{Oral dosing}

The different substances used for dosing were DM (99.5\%, Chem Service, West Chester, PA, USA), technical-grade DDT (98\%, Sigma-Aldrich, Steinheim, Germany), p-NP (Sigma-Aldrich) and phytoestrogens [Coumesterol: $\quad$ 95\% (Sigma-Aldrich), Genistein: 98\% (Sigma-Aldrich), (Zearalenone) Sigma-Aldrich]. 
The concentration of the different substances was obtained from reports in the literature and was then calculated according to exposure levels in South Africa. Cottonseed oil (Lot 21 K0162; Sigma-Aldrich) was used for the control group (Group A) as vehicle. Experimental Group B received $1.0 \mathrm{mg} \mathrm{kg}^{-1} \mathrm{DM}$, Group $C$ received a combination of $0.5 \mathrm{mg} \mathrm{kg}^{-1} \mathrm{DM}$ and $35 \mathrm{mg} \mathrm{kg}^{-1}$ DDT and Group D a combination of $0.5 \mathrm{mg} \mathrm{kg}^{-1} \mathrm{DM}, 35 \mathrm{mg} \mathrm{kg}^{-1}$ DDT, $2.5 \mu \mathrm{g} \mathrm{kg}^{-1} p-\mathrm{NP}, 2.5 \mu \mathrm{g} \mathrm{kg}^{-1}$ genistein, $2.5 \mu \mathrm{g} \mathrm{kg}^{-1}$ coumesterol and $2.5 \mathrm{\mu g} \mathrm{kg}^{-1}$ zearalenone. All the substances were administered intragastrically at a dose volume of $1 \mathrm{ml} \mathrm{kg}^{-1}$. The dosage volume for each animal was adjusted according to body mass.

\section{Observations and procedures}

Throughout the experiment, the animals were observed daily for clinical health, behavioural changes, signs of toxicity and mortality. They were weighed and dosed at the same time every day to exclude any external variables.

The weights of the F1 males were recorded before anaesthesia. The males were anaesthetised with Halothane (Safeline Pharmaceuticals (PTY) LTD, Wadeville, South Africa). Cardiac puncture was used for blood sample collection. The blood collection, together with an overdose of Halothane vapour was used for termination. The blood was spun down and the serum was stored at $-20{ }^{\circ} \mathrm{C}$ for liver function analysis $(n=6)$ and chemical analysis $(n=6)$.

After termination, the anogenital distance (AGD) was measured; the testes, epididymides, seminal vesicles and the liver were weighed and the right testis fixed for histological evaluation.

\section{Anogenital distance}

The AGD, the length of the perineum from the base of the genital tubercle to the centre of the anus when the skin was naturally extended without stretching, was measured with a ruler in millimeters (Fielden et al., 2002). The AGD was measured by the same individual to increase precision and to control for operator variation.

\section{Testes, epididymides, seminal vesicles}

The left and right testes and epididymides were removed and the epididymides were separated from the testes, cleaned and weighed individually. Both left and right seminal vesicles were removed and weighed. The mean testicular, epididymal and seminal vesicle mass were calculated. The right testis was placed in Bouins solution and was used for histology, with special reference to spermatogenesis (De Jager et al., 1999a,b).

\section{Cauda epididymal sperm count}

The left cauda epididymis was used to determine the sperm concentration. The cauda epididymis was removed by the same individual to increase precision and to control for operator variation. The cauda epididymis was separated from the caput-corpus and was placed in $2 \mathrm{ml}$ of phosphate buffered saline (PBS) medium (cat. no. BR14a, Oxiod, Hampshire, England) in a Petri dish. The cauda epididymis was cut into very small pieces to free the sperm. The PBS with sperm was transferred to a Falcon tube (De Jager et al., 1999a,b). The Neubauer method was used to determine the sperm concentration, expressed as million per millilitre (WHO, 1999)

\section{Testicular Histology and Staging}

The testicular samples were fixed in Bouins fixative and were washed with $70 \%$ ethanol to remove the fixative. Fixed cross-sections of the testes were embedded in paraffin wax and the testicular tissue was dehydrated in a graded series of ethanol. Thin sections, $3 \mu \mathrm{m}$, were cut on a microtome and stained with a modified periodic acid-Schiff's reaction (PAS) and counterstained with Alum haematoxylin.

These slides were used to do staging of spermatogenesis using a Nikon Optiphot photomicroscope with $10 \times$ and $100 \times$ objectives. A computer software program on spermatogenesis, stages ${ }^{\mathrm{TM}} 2.1$ (Vanguard 
Media Inc., IL, USA), aided in the staging process together with the histology atlas of Russell et al. (1990).

For each individual rat, 30 randomly selected seminiferous tubules were staged to identify and classify all 14 stages of spermatogenesis. The tubular diameter, seminiferous epithelium and lumen diameter for all 30 tubules were measured both horizontally and vertically. The mean values of the horizontal and vertical measurements for each parameter were used for statistical analyses of the tubular diameter, seminiferous epithelium and lumen diameter.

\section{Statistical analyses}

The Kruskal-Wallis one-way nonparametric analysis of variance (AOV) was used for the comparison of total body and liver weights. For between group comparisons of all other variables one-way anova (Analysis of variance) was performed using ranks followed by pair-wise comparisons with Fisher's LSD (least significant difference method). Pair-wise comparisons between the control group and the treatment groups were performed at the Bonferroni adjusted level of significance $(0.05 / 4=0.012)$ with the Wilcoxon Rank Sum test.

\section{Results}

No statistically significant differences were observed in total body weight between the groups. The general condition of the F1 males appeared to be unchanged. The parametric one-way anova performed on the ranked data followed by pair-wise comparisons with Fisher's LSD showed that the AGD of Groups $A$ and $B$, and the AGD of Groups $B, C$ and $D$ were not significantly different from one another. Pair-wise comparison between the experimental groups and the control group, using the Wilcoxon Rank Sum test, indicated that the AGD of Groups B $(P=0.047), C(P=0.045)$ and $D(P=0.002)$ were significantly shorter compared with the control group (Table 1).

Table 1 The effect of various admixtures on reproductive parameters of the F1 males of the different treatment groups

\begin{tabular}{|c|c|c|c|c|c|c|c|}
\hline & A & B & $A / B$ & C & $A / C$ & D & A/D \\
\hline Variables & $\begin{array}{l}\text { Median } \\
\text { (range) }\end{array}$ & $\begin{array}{l}\text { Median } \\
\text { (range) }\end{array}$ & $\begin{array}{c}P \text { - } \\
\text { value }\end{array}$ & $\begin{array}{l}\text { Median } \\
\text { (range) }\end{array}$ & $\begin{array}{c}P \text { - } \\
\text { value }\end{array}$ & $\begin{array}{l}\text { Median } \\
\text { (range) }\end{array}$ & $P$-value \\
\hline Body mass (g) & $\begin{array}{l}543.7 \\
(488.8- \\
649.3)\end{array}$ & $\begin{array}{l}538.7 \\
(472.0- \\
679.4)\end{array}$ & ns & $\begin{array}{l}526.7 \\
(475.7- \\
587.3)\end{array}$ & ns & $\begin{array}{l}561.2 \\
(500.8- \\
630.9)\end{array}$ & ns \\
\hline $\begin{array}{l}\text { Anogenital distance } \\
(\mathrm{mm})\end{array}$ & $55(50-60)$ & $52(38-56)$ & 0.047 & $51(42-58)$ & 0.045 & $50(42-56)$ & 0.002 \\
\hline $\begin{array}{l}\text { Seminal vesicle mass } \\
\text { (g) }\end{array}$ & $\begin{array}{l}0.637 \\
(0.404- \\
0.815)\end{array}$ & $\begin{array}{l}0.580 \\
(0.394- \\
0.654)\end{array}$ & 0.046 & $\begin{array}{l}0.615 \\
(0.464- \\
0.724)\end{array}$ & ns & $\begin{array}{l}0.667 \\
(0.481- \\
0.936)\end{array}$ & ns \\
\hline Epididymal mass (g) & $\begin{array}{l}0.661 \\
(0.615- \\
0.813)\end{array}$ & $\begin{array}{l}0.627 \\
(0.477- \\
0.710)\end{array}$ & ns & $\begin{array}{l}0.653 \\
(0.589- \\
0.668)\end{array}$ & ns & $\begin{array}{l}0.653 \\
(0.593- \\
0.786)\end{array}$ & ns \\
\hline Testicular mass (g) & $\begin{array}{l}1.986 \\
(1.777- \\
2.055)\end{array}$ & $\begin{array}{l}1.896 \\
(1.777- \\
2.055)\end{array}$ & ns & $\begin{array}{l}1.955 \\
(1.676- \\
2.143)\end{array}$ & ns & $\begin{array}{l}1.928 \\
(1.805- \\
2.329)\end{array}$ & ns \\
\hline $\begin{array}{l}\text { Seminiferous tubule } \\
\text { diameter }(\mu \mathrm{m})\end{array}$ & $\begin{array}{l}607.2 \\
(507.0- \\
695.1)\end{array}$ & $\begin{array}{l}544.6 \\
(440.7- \\
587.0)\end{array}$ & $<0.001$ & $\begin{array}{l}513.7 \\
(479.7- \\
550.7)\end{array}$ & $<0.001$ & $\begin{array}{l}518.1 \\
(393.8- \\
595.1)\end{array}$ & $<0.001$ \\
\hline
\end{tabular}




\begin{tabular}{|c|c|c|c|c|c|c|c|}
\hline & A & B & $A / B$ & C & $A / C$ & D & A/D \\
\hline Variables & $\begin{array}{l}\text { Median } \\
\text { (range) }\end{array}$ & $\begin{array}{l}\text { Median } \\
\text { (range) }\end{array}$ & $\begin{array}{c}P- \\
\text { value }\end{array}$ & $\begin{array}{l}\text { Median } \\
\text { (range) }\end{array}$ & $\begin{array}{c}P- \\
\text { value }\end{array}$ & $\begin{array}{l}\text { Median } \\
\text { (range) }\end{array}$ & $P$-value \\
\hline $\begin{array}{l}\text { Seminiferous } \\
\text { epithelium thickness } \\
(\mu \mathrm{m})\end{array}$ & $\begin{array}{l}57.39 \\
(43.63- \\
64.70)\end{array}$ & $\begin{array}{l}50.78 \\
(41.38- \\
54.16)\end{array}$ & 0.030 & $\begin{array}{l}42.36 \\
(36.23- \\
50.74)\end{array}$ & $<0.001$ & $\begin{array}{l}40.34 \\
(32.50- \\
55.69)\end{array}$ & $<0.001$ \\
\hline Lumen diameter $(\mu \mathrm{m})$ & $\begin{array}{l}88.32 \\
(63.71- \\
120.87)\end{array}$ & $\begin{array}{l}78.64 \\
(55.09- \\
105.48)\end{array}$ & ns & $\begin{array}{l}84.72 \\
(72.10- \\
113.78)\end{array}$ & ns & $\begin{array}{l}93.77 \\
(48.78- \\
123.88)\end{array}$ & ns \\
\hline $\begin{array}{l}\text { Total sperm count } \\
\left(\times 10^{6}\right)\end{array}$ & $\begin{array}{l}70.40 \\
(53.53- \\
103.13)\end{array}$ & $\begin{array}{l}64.53 \\
(27.00- \\
90.00)\end{array}$ & ns & $\begin{array}{l}54.16 \\
(27.00- \\
106.33)\end{array}$ & 0.013 & $\begin{array}{l}48.76 \\
(12.53- \\
68.80)\end{array}$ & 0.003 \\
\hline
\end{tabular}

The absolute testicular mass was reported rather than mass relative to body mass (testis ratio), as testicular mass and body mass are reported to be largely independent variables (Ettlin \& Dixon, 1985). The testicular masses of the experimental groups were not significantly different compared with those of the control group.

The mean epididymal mass of the experimental groups compared with the control group were also not significantly different.

Between groups comparison with the anova performed on ranked data showed that the seminal vesicle mass of Group B differed significantly from Group A and Group D. The Wilcoxon Rank Sum test indicated that the median seminal vesicle mass of Group B $(P=0.046)$ was significantly lower compared with Group A. Seminal vesicle masses of Groups C and D did not differ significantly from Group A (Table 1).

The spermatogenesis of most of the animals in the experimental groups was normal and all 14 stages of spermatogenesis were present. The rat in Group $C$ with the undescended testis had normal spermatogenesis in the right testis, but no spermatogenesis could be identified in the undescended testes. One rat in Group D had what looked like crystals inside the tubules and again no spermatogenesis could be observed.

Although the stages of the experimental groups were normal, there were signs of vacuolisation and cell necrosis with sloughing of the epithelium of the tubules of the experimental groups. The degree of vacuolisation and sloughing increased from Group B to Group D (Fig. 1).

When the anova was performed on the data using ranks followed by pair-wise comparisons with Fishers LSD, the diameters of all three experimental groups were observed to be smaller than those of the control group. Diameters of Groups C and D were also smaller than those of Group B. Groups C and D were not significantly different from one another. The Wilcoxon Rank Sum test showed that the tubule diameters of all experimental groups were significantly smaller compared with those of the control group (Table 1).

Application of the anova on ranked data of the seminiferous epithelium showed that all groups differed significantly from one another with the exception of Groups C and D, which had similar epithelial thicknesses. Paired comparisons using Wilcoxon Rank sum test between the experimental groups and 
the control group showed that the epithelial thickness of Groups B $(P=0.030), \mathrm{C}(P=<0.001)$ and $\mathrm{D}$ $(P=<0.001)$ was significantly thinner compared with that of Group A (Table 1$)$. No statistically significant difference was observed between the groups in measurements of the lumen diameter.
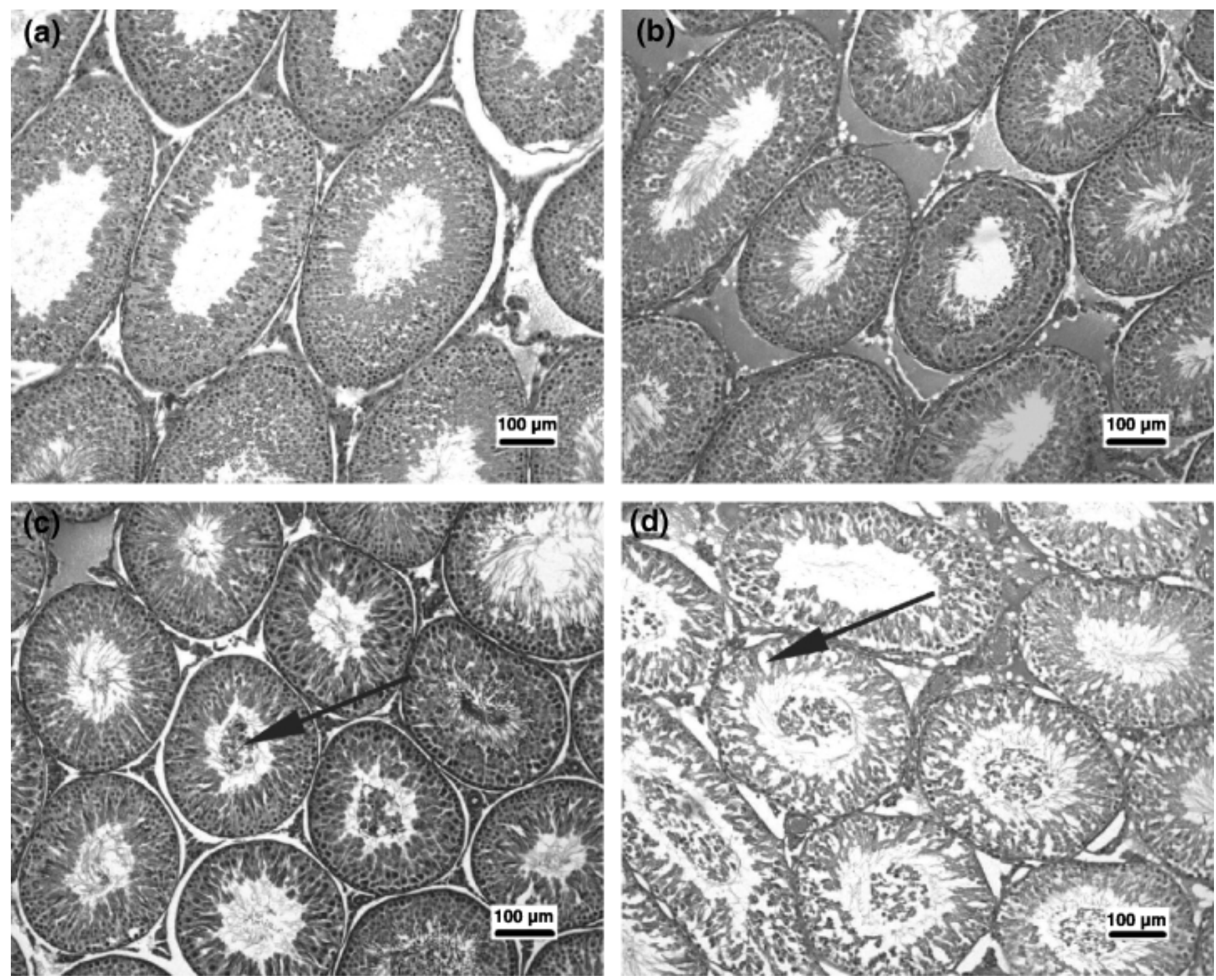

Fig. 1 Testicular histology. Note the increase in apical sloughing [indicated by the arrow in (c)] and vacuolisation [(indicated by die arrow in (d)] in the experimental groups. Group A = control; Group B = deltamethrin (DM); Group $C=D M+$ dichlorodiphenyltrichloroethane (DDT); Group $\quad D=D M+D D T+p$-nonylphenol (NP) + phytoestrogens modified periodic acid-Schiff's reaction (PAS) staining and counterstained with Alum hematoxylin; magnification, $\times 10$

The sperm count of Groups $C$ and $D$ were significantly lower compared with that of Group A. The total sperm count of Group A and B, and the total sperm count of Groups B, C and D were not significantly different from one another when comparisons were performed with the anova following ranking of the data. The Wilcoxon Rank Sum test indicated that the total sperm count of Group A was significantly higher than the total sperm count of Groups $C(P=0.013)$ and $D(P=0.003)($ Table 1$)$.

Comparison of liver function enzymes with one-way AOV applied on ranked data showed that serum ALT levels of Groups A, B, and C did not differ significantly, as was the case for Groups C and D (Table 2). Comparison with control group, using the Wilcoxon Rank Sum Test showed that Group D $(P=0.013)$ serum alanine aminotransferase (ALT) levels were significantly lower. Serum asparate aminotransferase (AST) levels of the four groups did not appear to differ significantly from one another. 
The Wilcoxon Rank Sum test indicated a significant increase in the liver mass between Groups $\mathrm{A}$ and $\mathrm{C}$ $(P=0.0019)$ and $\mathrm{A}$ and $\mathrm{D}(P=0.0001)$. The liver mass of Group B was not affected.

Table 2 The effect of various admixtures on the liver

\begin{tabular}{|c|c|c|c|c|c|c|c|}
\hline & A & B & $A / B$ & C & $A / C$ & D & $A / D$ \\
\hline Variables & $\begin{array}{l}\text { Median } \\
\text { (range) }\end{array}$ & $\begin{array}{l}\text { Median } \\
\text { (range) }\end{array}$ & $\begin{array}{c}P- \\
\text { value }\end{array}$ & $\begin{array}{l}\text { Median } \\
\text { (range) }\end{array}$ & $P$-value & $\begin{array}{l}\text { Median } \\
\text { (range) }\end{array}$ & $P$-value \\
\hline $\begin{array}{l}\text { Serum } \\
\text { ALT (IU/I) }\end{array}$ & $\begin{array}{l}58.35 \\
(49.90- \\
61.90)\end{array}$ & $\begin{array}{l}57.05 \\
(43.30- \\
63.80)\end{array}$ & ns & $\begin{array}{l}4 \times .15 \\
(36.50- \\
47.30)\end{array}$ & ns & $\begin{array}{l}37.25 \\
(26.80- \\
50.60)\end{array}$ & $P=0.013$ \\
\hline $\begin{array}{l}\text { Serum } \\
\text { AST (IU/I) }\end{array}$ & $7.2(3.6-9.7)$ & $\begin{array}{l}6.10(4.30- \\
16.80)\end{array}$ & ns & $\begin{array}{l}7.20(5.23- \\
9.00)\end{array}$ & ns & $\begin{array}{l}9.45(8.20- \\
16.00)\end{array}$ & ns \\
\hline $\begin{array}{l}\text { Liver mass } \\
\text { (g) }\end{array}$ & $\begin{array}{l}20.68 \\
(19.00- \\
23.28)\end{array}$ & $\begin{array}{l}20.00 \\
(17.06- \\
28.39)\end{array}$ & ns & $\begin{array}{l}25.57 \\
(21.92- \\
26.96)\end{array}$ & $P=0.013$ & $\begin{array}{l}27.20 \\
(24.34- \\
29.62)\end{array}$ & $P=0.005$ \\
\hline
\end{tabular}

$\mathrm{A}$, control; $\mathrm{B}$, deltamethrin (DM); $\mathrm{C}, \mathrm{DM}+$ dichlorodiphenyltrichloroethane (DDT); $\mathrm{D}, \mathrm{DM}+\mathrm{DDT}+p$ nonylphenol (NP) + Phytoestrogens; ns, nonsignificant.

For the chemical analysis of the blood only DDT and both its metabolites (DDE and DDD) were found in Groups $C$ and $D$ with the limit of detection set at $40 \mu \mathrm{g} \mathrm{I}^{-1}$ blood. None of the other drugs were above the detection limit.

\section{Discussion}

This study investigated the endocrine disrupting effects of in utero, lactational and direct exposure to DM, a combination of DM and DDT and a combination of DM, DDT, $p$-NP and phytoestrogens on the liver and on the reproductive parameters of the F1 male rats.

The AGD of all three experimental groups were significantly shorter compared with the control group. Reduced male AGD is an indication of feminisation and has been observed after treatment with antiandrogenic and oestrogenic compounds (Gray et al., 1994; Kelce et al., 1994). The degree by which the AGD were affected increased per group as more compounds were added to the mixture. It has not yet been proven whether DM has an anti-androgenic effect (Andrade et al., 2002), but the persistent DDT metabolite $p, p^{\prime}$-DDE is well known for its anti-androgenic activity (Kelce et al., 1995; Gray et al., 1999). On the other hand, DDT as well as the phytoestrogens and $p$-NP exerts oestrogenic activity and it has been shown that low doses of $0, p^{\prime}$-DDT are associated with a significant decrease in the AGD (Palanza et al., 2001). This might therefore be an oestrogenic effect rather than an anti-androgenic effect.

Although the epididymal mass of the experimental groups were not affected, it is possible that the histology and cell function of this androgen-dependent organ were perturbed. The seminal vesicle mass of Group B was significantly lower compared with that of the control group. During developmental stages, adequate concentrations of androgens are necessary for the accessory sex organs to develop normally (Mably et al., 1992). DM is known to have oestrogenic properties and El-Aziz et al. (1994) also found impaired reproductive function in laboratory animals at $1 \mathrm{mg} \mathrm{kg}^{-1}$.

The absolute testicular masses of the experimental groups did not differ statistically significantly from those of the control group. Although the testicular masses of the experimental groups do not appear to be affected, the testicular histology demonstrates significant effects. Except for the lumen diameter, both 
the seminiferous tubule diameter and the seminiferous epithelium thickness were significantly different compared with the control group. As with the AGD, Groups C and D appeared to be more affected than Group B. This may be indicative of testicular toxicity at the dose levels tested (De Jager et al., 1999a; Andrade et al., 2002).

All three experimental groups showed signs of Sertoli cell toxicity as became evident in some animals as germ cell necrosis, apical sloughing and vacuolisation (Russell, 1993). EDCs can reduce the size of the seminiferous tubules and thereby decrease the number of Sertoli cells. The number of Sertoli cells present during the prepubertal period is responsible for orchestrating and regulating spermatogenesis later in adulthood (Sharpe \& Skakkebæk, 1993). Furthermore, Sertoli cell multiplication is controlled to a large extent by follicle-stimulating hormone (FSH) (Gustafson \& Donahoe, 1994). Because oestradiol exerts negative feedback on FSH secretion, exposure to oestrogenic compounds during male sexual development could substantially reduce FSH secretion, reducing Sertoli cell multiplication (Cheek \& McLanchlan, 1998). The consequences for the adult male would include decreased sperm production and, potentially, cryptorchidism (Sharpe \& Skakkebæk, 1993). It is therefore not surprising that the mean total cauda epididymal sperm count of the experimental groups was lower compared with that of the control group. Although the sperm count of Group B was not significantly lower than the control group, those of Groups C and D were. Reduced cauda epididymal sperm number is a sensitive and persistent indicator of in utero and lactational anti-androgen exposure. DDT was used in both Group C and D and the anti-androgenic activity of the persistent DDT metabolite, $p, p^{\prime}$-DDE has been demonstrated by Kelce et al. (1995) and Gray et al. (1999).

The substances and combinations of the substances used in Groups C and D are clearly hepatotoxic as indicated by the increased liver weights, which were significantly higher than that in the control group. DDE induces cytochrome P450 in the liver (You et al., 1999). This induction of enzymes may account for the increased liver weight in the exposed animals (You et al., 1999; Leavens et al., 2002). Nagao et al. (2001) found that long-term administration of $p$-NP at $50 \mathrm{mg} \mathrm{kg}^{-1}$ day $^{-1}$ has a toxic effect on the liver of adult rats. However, at lower concentrations $\left(2\right.$ and $10 \mathrm{mg} \mathrm{kg}^{-1}$ day $\left.^{-1}\right)$ they found no effect on the liver. In this study, the concentrations of DDT and $p$-NP that were used in Groups C and D, were lower than concentrations that were used by the above-mentioned investigators; it is however the group that was affected the most. The decrease in serum ALT in Groups $C$ and $D$ may possibly be due to oxidative stress (Lee \& Jacobs, 2006).

The effect of mixtures and low doses became evident in this study. If the results of Group C to Group D are compared, it is alarming to see the difference in testicular histology, AGD, sperm count and liver weight when phytoestrogens and $p$-NP were added to the mixture of DDT and DM in $\mathrm{gg} \mathrm{kg}^{-1}$. These results confirmed Payne et al. (2000) finding that substances in mixtures act additively even when they are present at concentrations that would have individually produced no detectable effects. This study further provides evidence that exposure of rats to a relevant environmental mixture of known endocrine disrupting substances during gestation, lactation and direct exposure up to 14 weeks, has a profoundly detrimental influence on male reproductive parameters.

People living in malaria stricken areas in South Africa and other countries, where spraying with DDT forms part of the malaria prevention protocol, may readily be exposed to all of the substances tested in this study. Although none of the animals died as a result of exposure to the combination of these substances, the reproductive system of the males were adversely affected. It is of the utmost important to realise that DDT in combination with low doses of other EDCs may be harmful to wildlife and to humans. These findings are also consistent with what was found in epidemiological studies in Mexico (De Jager et al., 2006) and South Africa (Aneck-Hahn et al., 2007) where young men were exposed to DDT. It is imperative to investigate the effects of these EDCs at different concentrations and in other combinations on male reproductive health in animals and in humans. 


\section{Acknowledgements}

We would like to thank the University of Pretoria Biomedical Research Centre, in particularly Mr P. Selahle, and the staff of the Andrology laboratory, University of Pretoria, for their technical support. The South African National Research Foundation (NRF) funded this project.

\section{References}

AndradeAJ, AraujoS, SantanaGM, OhiM, DalsenterPR (2002) Reproductive effects of deltamethrin on male offspring of rats exposed during pregnancy and lactation. Regul Toxicol Pharmacol 36:310-317.

Aneck-HahnNH, SchulenburgGW, BornmanMS, FariasP, De JagerC (2007) Impaired semen quality associated with environmental DDT exposure in young men living in a malaria area in the Limpopo Province, South Africa. $J$ Androl 28:423-434.

BarnhoornIEJ, BornmanMS, DreyerL, de JagerC (2002) Testicular calcifications in the common eland (Tragelapus oryx): a possible link to endocrine disruption? Paper presented at the 9th International Symposium on Spermatology. Cape Town, South Africa, 6-11 October 2002.

BouwmanH, SeredaB, MeinhardtHM (2006) Simultaneous presence of DDT and pyrethroid residues in human breast milk from a malaria endemic area in South Africa. Environ Poll 144:902-917.

CharlesGD, KanHL, SchislerMR, Bhaskar GollapudiB, Sue MartyM (2005) A comparison of in vitro and in vivo EDSTAC test battery results for detecting antiandrogenic activity. Toxicol Appl Pharmacol 202:108-120.

CheekAO, McLanchlanJA (1998) Environmental hormones and the male reproductive system. J Androl 19:5-10.

ChenH, XiaoJ, HuG, ZhouJ, XiaoH, WangX (2002) Estrogenicity of organophosphorus and pyrethroid pesticides. J Toxicol Environ Health A 65:1419-1435.

De JagerC, BornmanMS, Van der HorstG (1999a) I. The effect of p-nonylphenol, an environmental toxicant with oestrogenic properties, on fertility potential in adult male rats.. Andrologia 31:99-106.

De JagerC, BornmanMS, OosthuizenJMC (1999b) II. The effect of $p$-nonylphenol on the fertility potential of male rats after gestational, lactational and direct exposure. Andrologia 31:107-113.

De JagerC, MyburghJ, van der BurgB, LemmenJG, BornmanMS (2002) Estrogenic contamination of South African river waters: a pilot study. Endocrine Disruptor and the Water Industry Symposium. Cincinnati, Ohio, USA, April 1820, 2002.

De JagerC, FariasP, Hernandez AvillaM, BarrazaA, Diaz SanchezV, CerezoG, AyotteP, DewaillyÉ, BaileyJL (2006) Reduced seminal parameters associated with environmental DDT exposure in men from Chiapas, Mexico. J Androl $27: 16-27$.

DiakaJK, OdriguesR, GoudazeG (1998) Influence of genistein (4',5',7'-trihydroxy-isoflavone) on the growth and proliferation of testicular cell lines. Biol Cell 90:349-354.

El-AzizAMI, SahlabAM, El-KhalikAM (1994) Influence of diazinon and deltamethrin on reproductive organs and fertility of male rats. Dtsch Tierarztl Wochenschr 101:230-232.

EttlinRA, DixonRL (1985) Reproductive toxicity. In: Environmental Pathology. MottetNK (ed). Oxford University Press, New York, pp. 129-180.

FacemireCF, GrossTS, MassonGR, et al. (1995) Reproductive impairment in the Florida panther: nature of nurture? Environ Health Perspect 103:79-86. 
FieldenMR, HalgrenRG, FongCJ, StaubC, JohnsonL, ChouK, ZacharewskiTR (2002) Gestational and lactational exposure of male mice to diethylstilbestrol causes long-term effects on the testis, sperm fertilizing ability in vitro, and testicular gene expression. Endocrinology 143:3044-3059.

GoV, GareyJ, WolffMS, PogoBGT (1999) Estrogenic potential of certain pyrethroid compounds in the MCF-7 human breast carcinoma cell line. Envrion Health Perspect 107:173-177.

GrayLEJr, FerrellJ, OstbyJ, RehnberG, LinderR, CooperR, GoldmanJ, SlottV, LaskeyJ (1989) A dose response analysis of methoxychlor-induced aterations of reproductive development and function in the rat. Fundam Appl Toxicol 12:92-108.

GrayLEJr, OstbyJS, KelceWR (1994) Developmental effects of an envrionmental antiandrogen: the fungicide vinclozolin alters sex differentiation of the male rat. Toxicol Appl Pharmocol 129:46-52.

GrayLE, OstbyJ, MonossonE, KelceWR (1999) Environmental antiandrogens: low doses of the fungicide cinclozolin alter sexual differentiation of the male rat. Toxicol Indus Health 15:48-64.

GrayLEJr, OstbyJ, FurrJ, et al. (2001) Effects of environmental androgens on reproductive development in experimental animals. Hum Reprod Update 7:248-264.

GustafsonML, DonahoePK (1994) Male sex determination: current concepts of male sex differentiation. Annu Rev Med 45:504-524.

HargreavesK, KoekemoerLL, BrookBD, HuntRH, MthembuJ, CoetzeeM (2000) Anopheles funestus resistant to pyrethroid insecticides in South Africa. Med Vet Entomol 14:181-189.

JensenTK, ToppariJ, KeidingN, SkakkebaekNE (1995) Do environmental estrogens contribute to the decline in male reproductive health? Clin Chem 41:1896-1901.

KelceWR, MonossonE, GamcsikMP, LawsSC, GrayLE (1994) Environmental hormone disrupters: evidence that cinclozolin developmental toxicity is mediated by antiandrogenic metabolites. Toxicol Appl Pharmacol 126:276285.

KelceWR, StoneCR, LawsSC, GrayLEJr, KemppainenJA, WilsonEM (1995) Persistent DDE metabolite $p, p$ '-DDE is a potent androgen receptor antagonist. Nature 375:581-585.

LeavensTL, SparrowBR, DevitoMJ (2002) Lack of antiandrogenic effects in adult male rats followung acute exposure to 2,2-bis(4-chlorophenyl)-1,1-dichloroethylene (p,p'-DDE). Toxicol 174:69-78.

LeeD-H, JacobsDRJr (2006) Association between serum concentrations of persistent organic pollutants and $\mathrm{Y}$ glutamyltransferase: results from the National Health and Examination Survey 1999-2002. Clin Chem 52:18251827.

LeePC, ArndtP, NickelsKC (1999) Testicular abnormalities in male rats after lactational exposure to nonylphenols. Endocrine 11:61-68.

MablyTA, MooreRW, PetersonRE (1992) In utero and lactational exposure of male rats to 2,3,7,8tetrachlorodibenzo-p-dioxin. Toxicol Appl Pharmacol 114:97-107.

NagaoT, KazuyoshiW, MarumoH, YoshimuraS, OnoH (2001) Reproductive effects of nonylphenol in rats after gavage administration: a two-generation study. Repro Toxic 15:293-315.

OECD Guidelines for the Testing of Chemicals (1983). One-generation reproductive toxicity study (Protocol 415). 26 May 1983, pp. 1-8.

PalanzaP, ParmigianiS, vom SaalFS (2001) Effects of prenatal exposure to low doses of diethylstilbestrol, $o, p$ 'DDT, and methoxychlor on postnatal growth and neurobehavioral development in male and female mice. Horm Behav 40:252-265.

PayneJ, RajapakseN, WilkinsM, KortenkampA (2000) Prediction and assessment of the effects of mixtures of four xenoestrogens. Environ Health Perspect 108:983-987. 
RussellLD (1993) Morphological and functional evidence for Sertoli-germ cell relationships. In: The Sertoli Cell. RussellLD, GriswoldMD (eds). Cache River Press, Clearwater, pp. 365-390.

RussellLD, EttlinRA, HikimAPS, CleggED (1990) Histological and histopathological evaluation of the testis. Cache River Press, Clearwater.

SanttiR, MäkeläS, StraussL, KorkmanJ, KostianM-L (1998) Phytoestrogens: potential endocrine disruptors in males. Toxicol Indus Health 14:223-237.

SharpBL, le SueurD (1996) Malaria in South Africa - the past, the present and selected implications for the future. S Afr Med J 86:83-89.

SharpeRM, SkakkebækNE (1993) Are estrogens involved in falling sperm counts and disorders of the male reproductive tract? Lancet 341:1392-1395.

SumpterJP (1995) Feminized responses in fish to environmental estrogens. Toxicol lett 82 83:737-742.

ToppariJ, LarsenJC, ChristiansenP, GiwercmanA, GrandjeanP, GuilletteLJJr, JégouB, et al. (1996) Male reproductive health and environmental xenoestrogens. Environ Health Perspect 104:741-803.

TurusovV, RakitsskyV, TomatisL (2002) Dichlorodiphenylchloroethane (DDT): ubiquity, persistence, and risk. Environ Health Perspect 110:125-128.

World Health Organization (WHO) (1999) Laboratory Manual for the Examination of Human Semen and SpermCervical Mucus Interaction, 4th edn. Cambridge University Press,

New York, NY.

YouLi, Brenneman KarrieA, Heck Henry d'A (1999) In utero exposrue to antiandrogens alters the responsiveness of the prostate to p,p'-DDE in adult rats and may induce prostatic inflammation. Toxicol App Pharmacol 161:258266. 